\title{
Measure distorted arrival rate risks and their rewards
}

\author{
Dilip B. Madan
}

Received: 16 January 2017 / Accepted: 2 June 2017 / Published online: 26 June 2017

(C) The Author(s). 2017 Open Access This article is distributed under the terms of the Creative Commons Attribution 4.0 International License (http://creativecommons.org/licenses/by/4.0/), which permits unrestricted use, distribution, and reproduction in any medium, provided you give appropriate credit to the original author(s) and the source, provide a link to the Creative Commons license, and indicate if changes were made.

\begin{abstract}
Risks embedded in asset price dynamics are taken to be accumulations of surprise jumps. A Markov pure jump model is formulated on making variance gamma parameters deterministic functions of the price level. Estimation is done by matrix exponentiation of the transition rate matrix for a continuous time finite state Markov chain approximation. The motion is decomposed into a space dependent drift and a space dependent martingale component. Though there is some local mean reversion in the drift, space dependence of the martingale component renders the dynamics to be of the momentum type. Local risk is measured using market calibrated measure distortions that introduce risk charges into the lower and upper prices of two price economies. These risks are compensated by the exponential variation of space dependent arrival rates. Estimations are conducted for the S\&P 500 index (SPX), the exchange traded fund for the financial sector $(X L F)$, J. P. Morgan stock prices $(J P M)$, the ratio of $J P M$ to $X L F$, and the ratio of $X L F$ to $S P X$.
\end{abstract}

Keywords Variance Gamma $\cdot$ Hunt process - Markov chain approximation · Matrix exponentiation $\cdot$ Momentum function $\cdot$ Measure distortion

JEL Classification $\mathrm{C} 13 \cdot \mathrm{C} 14 \cdot \mathrm{G} 1 \cdot \mathrm{G} 10 \cdot \mathrm{G} 12$

\section{Introduction}

Asset prices in financial markets, following Madan and Schoutens (2016b), must change of necessity to support the price with a return for the investor. Furthermore, the changes must be surprises, if all available information has already entered the

D.B. Madan $(\bowtie)$

Robert H. Smith School of Business, University of Maryland, College Park, MD 20742, USA

e-mail: dbm@rhsmith.umd.edu 
price. As a consequence the changes must occur at surprise times. The simplest model for such times are the arrival times of a Poisson process. The price process is then rendered to be a pure jump process with no continuous component, either deterministic or random. The process for the logarithm of the price is therefore described by the structure for the arrival rates of jumps for all jump sizes $x,-\infty<x<\infty$. This arrival rate function may be denoted by $k(x, t)$ where the dependence on $t$ is in all generality, adapted to all information available at time $t$.

It was noted in Madan and Schoutens (2016b) that when the local motion is described by the generalized class of limit laws (Khintchine 1938; Lévy 1937; Sato 1999), of which the Gaussian law is an example, that then, in the non-Gaussian limit cases, the integral of the arrival rate function must be infinite. Such processes were termed infinite activity processes in Carr et al. (2002). The random variables at a finite horizon with arrival rate functions, that only depend on the jump size $x$, are then self-decomposable laws (Sato 1999). The models investigated in the paper are infinite activity processes.

It is then possible for the small jumps to be so frequent as they get smaller that the sum of all the small positive jumps is infinite and the sum of all the small negative jumps is negative infinity. Such processes are called processes of infinite variation. On the contrary, for finite variation processes the sum of all the small positive and negative jumps are separately finite, in any interval. More generally one may take the sum of all positive and negative jumps and their squares to be finite, and not just the small ones. These considerations require that

$$
\int_{-\infty}^{\infty} x k(x, t) d x<\infty ; \int_{-\infty}^{\infty} x^{2} k(x, t) d x<\infty .
$$

The processes being considered for the logarithm of the price are then infinite activity processes of finite variation and quadratic variation.

The use of an infinite activity process is motivated by recognizing that though the number of price moves in reality will be finite it is often quite large for daily data. Such observations inspire the use of limit laws. The limit laws are a special case of infinitely divisible laws termed self decomposable laws with a special structure to their arrival rate functions (Sato 1999). The arrival rate functions when scaled by the absolute jump size must be decreasing functions of the absolute jump size. In particular they are all infinite activity processes.

Finite quadratic variation is a consequence of working with semimartingales that is a maintained hypothesis for arbitrage free price dynamics (Delbaen and Schcharemayer 1994). Finite variation is a simplification that allows one to describe the price process as the difference of two increasing processes, one for the price up ticks and the other for the down ticks. In addition there is considerable evidence (see for a recent example Madan 2016c) that such processes are empirically adequate for describing the physical as well as the risk neutral process.

The stock price process $S(t)$, with $X(t)=\ln (S(t))$ then satisfies

$$
\begin{aligned}
X(t) & =X(0)+\sum_{s \leq t} \Delta X(s) \\
\Delta X(s) & =X(s)-X\left(s_{-}\right) .
\end{aligned}
$$


Additionally, associated with such a jump process is an integer valued random measure $\mu(d x, d t)$ that counts the jumps occurring in measurable subsets of space and time. One may then write

$$
\begin{aligned}
X(t) & =(x * \mu)_{t} \\
& =\int_{0}^{t} \int_{-\infty}^{\infty} x \mu(d x, d s) .
\end{aligned}
$$

The counting measure $\mu$ has a compensator $v(d x, d t)$ announcing the arrival rate for jumps at all times that is supposed to be defined by the arrival rate function $k(x, t)$ where

$$
v(d x, d t)=k(x, t) d x d t .
$$

All information about the log price process is then embedded in the arrival rate function and all modeling and estimation efforts are directed to the specification and estimation of arrival rate functions.

In particular there is no room for modeling drifts with or without mean reversion as the price process has no purely deterministic time component. Furthermore, there is also no room for a diffusive volatility given that there is no continuous martingale component in the price process. At the instantaneous level one may only speak of variation and quadratic variation but not directly of drift and volatility. Madan and Schoutens (2016b) shows that the limit of the continuously compounded drift over a horizon $h$, denoted by say $m_{t}(h)$ tends on division by $h$, to the exponential variation. Specifically, it may be observed that

$$
\lim _{h \rightarrow 0} \frac{m_{t}(h)}{h}=\int_{-\infty}^{\infty}\left(e^{x}-1\right) k(x, t) d x .
$$

The concept of reward compensating for risk defined in terms of arrival rate functions is then the exponential variation.

Madan and Schoutens (2016b) model the risk of holding stock positions by an assessment of their necessary fluctuations. The view taken is that prices must move to afford positions with a return and the risk is then that of how far up and down they may go. A temporally conservative evaluation of the magnitude of motion in both directions is constructed from the upper and lower prices prevailing in two price economies that not only exclude arbitrage but also eliminate highly acceptable trades.

The acceptable trades may be defined following Artzner et al. (1999) by a convex cone containing the nonnegative random variables. Every such cone may equivalently be defined by the class of random variables with a nonnegative expectation under a convex collection of test probability measures. The upper and lower prices are then suprema and infima of expectations under all test probabilities (see for example Cherny and Madan (2010), also Madan and Schoutens 2016a). Modeling the test probabilities as those delivering event probabilities bounded above by a concave distribution function evaluated at the physical event probability, leads to upper and lower prices as distorted expectations. The upper/lower distorted expectations (Kusuoka 2001) evaluate expectations using the distorted distribution function obtained by composing a convex/concave distribution function with the physical distribution function of the random variable in question. 
Risk exposures are then measured by the gap between the upper price and the expectation and the expectation less the lower price. Parametric distortions are employed with parameters calibrated to enforce the absence of acceptable opportunities in the options market for the S\&P 500 index. The convex and concave distorted physical distribution functions then sandwich the risk neutral distribution of the S\&P 500 index. In the continuous time limit probability distortions may be replaced by measure distortions (Madan et al. 2016) of arrival rate functions to construct upper and lower prices and their associated risk exposures. Such option calibrated risk exposures are then observed to be compensated by observed exponential variations across a wide variety of stock days. The result is an alternative asset pricing theory based on risk premia for two sided price fluctuations in opposition to classical covariation principles.

Efficient frontiers for risk and reward in terms of multidimensional arrival rate functions are presented in Madan (2016a). Optimal portfolios are here based on maximizing the lower price of the portfolio. This lower price may also be written as the exponential variation, seen as the reward, less the risk measured by the upper price for the centered and negated risk exposure. The continuous time centering is accomplished by subtracting the exponential variation.

Questions about the presence of local mean reversion or momentum in price processes are then to be addressed and answered by modeling and estimating arrival rate functions. Such considerations lead us to write arrival rate functions that depend on both the space variable $X$ and the jump size $x$. The data set on which the models will be estimated are short horizon returns from one to, say, five or ten days and hence time homogeneous models with arrival rate functions that do not specifically depend on calendar time are considered. Pure jump Markov processes with arrival rate functions depending on the space variable are called Hunt processes (Hunt 1966). They were formulated and estimated risk neutrally in Madan (2016b) using data on option prices. By way of contrast, the objective of this paper is on their estimation as a physical process from time series data. Mean reversion and momentum are important aspects of price dynamics influencing responses to abnormal price movements. With strong mean reversion one would sell on an extraordinary uptick while with momentum the appropriate action would be to buy on such upticks. Furthermore the structure of mean reversion and momentum can be different with respect to upward and downward moves and this is commented on in greater detail later. For examples of other applications of Hunt processes in the literature we cite Cont and Minca (2013) and Cousin et al. (2012).

Local mean reversion and momentum may be evaluated in the first instance by determining the effects on drift of down and up moves. Positive drifts associated with down moves and vice versa for the up moves represent mean reversion. The opposite result constitutes momentum. Beyond the drift one may address the impact on conditional probabilities of up moves of a given size conditional on such a move. If the conditional probability falls after such an up move then one has mean reversion upwards while if it rises then there is momentum upwards. Similar evaluations may be made for mean reversion and momentum downwards. This second approach was employed in Madan (2016b) to address mean reversion and momentum of risk neutral martingales where these aspects are absent in risk neutral drifts by construction. 
The outline of the rest of the paper is as follows. "Spatially inhomogeneous variance gamma processes" section introduces the spatially inhomogeneous variance gamma process to be estimated. "Estimation on time series data" section presents two estimation procedures based on matching digital moments and maximum likelihood. Results are presented for a variety of asset price time series in "Digital moment estimation result" section. "Drift structure of SIVG" section discusses the structure of mean reversion and momentum in the implied drift structure. "Mean reversion and momentum in the process" section reports on the structure of momentum and mean reversion for the process in aggregate, combining the drift and the martingale components. "Risk compensation" section reports on the implicit risk reward relationships. "The S\&P 500 index during the financial crisis" section presents results for the S\&P 500 index for the period of the financial crisis covering the years 2007 to 2009. "Conclusion" section concludes this paper.

\section{Spatially inhomogeneous variance gamma processes}

The variance gamma process $X(t)$ (Madan and Seneta 1990; Madan et al. 1998) was originally defined as a Brownian motion with drift $\theta$ and volatility $\sigma$ time changed by a gamma process with unit mean rate and variance rate $v$. For a standard Brownian motion $W(t)$ and a gamma process $g(t ; v)$ the variance gamma process with parameters $\sigma, v, \theta$ is

$$
X(t ; \sigma, v, \theta)=\theta g(t ; v)+\sigma W(g(t ; v))
$$

The gamma process is a positive increasing process with independent and identically distributed increments over nonoverlapping intervals of length $h$ that are gamma distributed with density

$$
f(x)=\frac{1}{v^{\frac{h}{v}} \Gamma\left(\frac{h}{v}\right)} x^{\frac{h}{v}-1} \exp \left(-\frac{x}{v}\right) .
$$

The variance gamma process is a Lévy process with characteristic function

$$
E[\exp (i u X(t))]=\left(\frac{1}{1-i u \theta v+\frac{\sigma^{2} v}{2} u^{2}}\right)^{\frac{t}{v}}
$$

It is a pure jump process with Lévy density $l(x)$ parameterized by $C, G, M$ (Carr et al. 2002) with

$$
l(x)=\frac{C}{|x|}\left(\mathbf{1}_{x<0} \exp (-G|x|)+\mathbf{1}_{x>0} \exp (-M x)\right),
$$


where

$$
\begin{aligned}
C & =\frac{1}{v} \\
G & =\frac{1}{\sigma} \sqrt{\left(\frac{2}{v}+\frac{\theta^{2}}{\sigma^{2}}\right)}+\frac{\theta}{\sigma^{2}} \\
M & =\frac{1}{\sigma} \sqrt{\left(\frac{2}{v}+\frac{\theta^{2}}{\sigma^{2}}\right)}-\frac{\theta}{\sigma^{2}} .
\end{aligned}
$$

The variance gamma process is a process of finite variation and it can be written as the difference of two increasing process.

The spatially inhomogeneous variance gamma process $(S I V G)$ is constructed by making the parameters of the variance gamma process in the $C G M$ parameterization deterministic functions of the space variable to form the jump compensator

$$
\begin{aligned}
v_{X_{t}}(d x, d t) & =k\left(X_{t}, x\right) d x d t \\
& =\frac{C\left(X_{t}\right)}{|x|}\left(\mathbf{1}_{x<0} \exp \left(-G\left(X_{t}\right)|x|\right)+\mathbf{1}_{x>0} \exp \left(-M\left(X_{t}\right) x\right)\right)
\end{aligned}
$$

for uniformly bounded and positive deterministic functions $C(X), G(X)$, and $M(X)$.

For details on the existence and uniqueness of the spatially inhomogeneous variance gamma process as a solution to the associated martingale problem described in Stroock (1975) and Stroock and Varadhan (1979), we refer the reader to Angelos (2013). Angelos (2013) follows Bass (1988) to demonstrate the existence and uniqueness of the spatially inhomogeneous variance gamma process.

The specific bounded functions employed, accommodate a local linearity around the current level tapering off to fixed levels at the two extremes. This is accomplished by the use of logistic functions of the form

$$
Y(X)=L+\frac{R-L}{1+\exp (-\eta X)}
$$

with parameters $L, R$, for the left and right limits and $\eta$ a speed of adjustment. There are then three parameters in each of the three deterministic functions $C(X), G(X)$, and $M(X)$ that we denote by $C_{L}, C_{R}, C_{\eta}, G_{L}, G_{R}, G_{\eta}, M_{L}, M_{R}$, and $M_{\eta}$. The result is a nine parameter spatially inhomogeneous Markov process for the evolution of jumps in the logarithm of the prices.

Defining

$$
\delta(X)=\int_{-\infty}^{\infty} x k(X, x) d x
$$

and $\mu_{X}(d x, d t)$ as the random measure with compensator $v_{X}(d x, d t)$, the process for $X(t)$ may also be written as

$$
d X=\delta(X) d t+d\left[x *\left(\mu_{X}-v_{X}\right)\right]_{t}
$$

with a space dependent $\operatorname{drift} \delta(X)$ and driven by the compensated jump martingale

$$
M(t)=\left[x *\left(\mu_{X}-v_{X}\right)\right]_{t} .
$$


Apart from mean reversion appearing via the implied drift $\delta(X)$, there may be mean reversion or momentum upwards or downwards depending on the dependence of $G$ and $M$ on $X$ as well as the presence of leverage via the dependence of $C$ on $X$. We present graphs comparing a Lévy process to one that endogenized leverage by raising $C$ on the down side relative to the up side. For mean reversion and momentum up and down the structure of $M$ and $G$ responses to the level of the process may be accordingly adjusted. For mean reversion up, $M(X)$ must rise with $X$ and fall with $X$ to have momentum up. Similarly for the downside and the dependence of $G$ on $X$.

Figure 1 presents a graph for the quarterly return density endogenizing leverage. Figure 2 presents the four cases of mean reversion down and up, momentum down and up, mean reversion down with momentum up, and momentum down with mean reversion up. The construction of the densities is described in the next section.

\section{Estimation on time series data}

For the estimation of a spatially inhomogeneous variance gamma process using data on the daily time series of prices, one needs to access the probabilities of returns over various horizons in the model. In this regard, we follow Pistorius and Mijatovic (2011) who show how to approximate a Lé vy process by a continuous time finite state Markov chain and use the approximation to price barrier options. They develop a matrix representation for the infinitesimal generator which on time scaling and matrix exponentiation delivers transition probability elements for the time scales involved. The states of the chain are constructed using a non-uniform grid. The generator is built by integrating the Lévy density between jumps sizes represented by the intergrid points for the large moves away from the immediate grid on the two sides of a current grid point. The transition rates to the immediate neighbours are built by a diffusion approximation. In the spatially inhomogeneous case considered here, we have a relevant Lé vy density at each grid point with parameters that vary with the

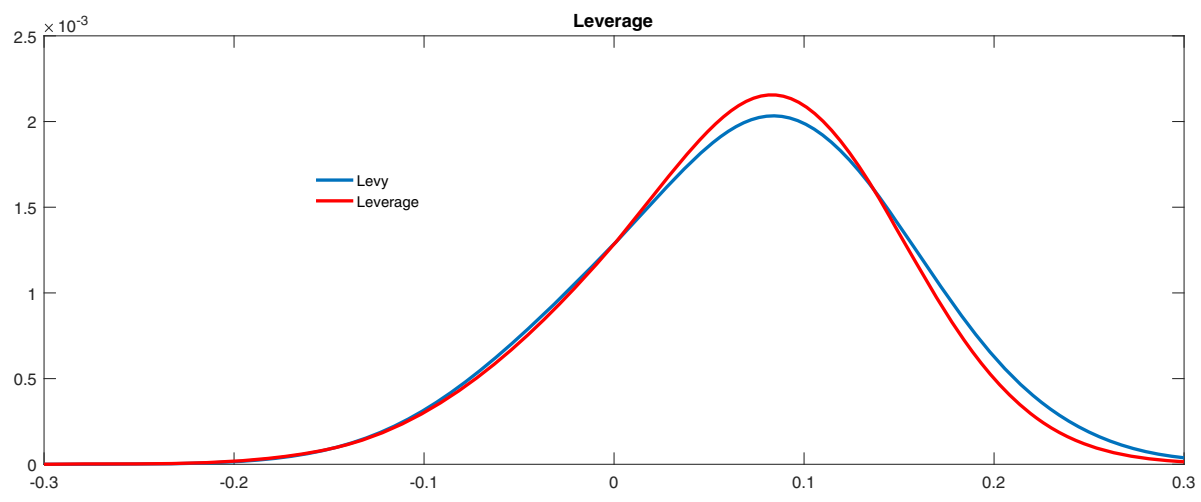

Fig. 1 Quarterly return densities with Leverage contrasted with that from a Lévy process 

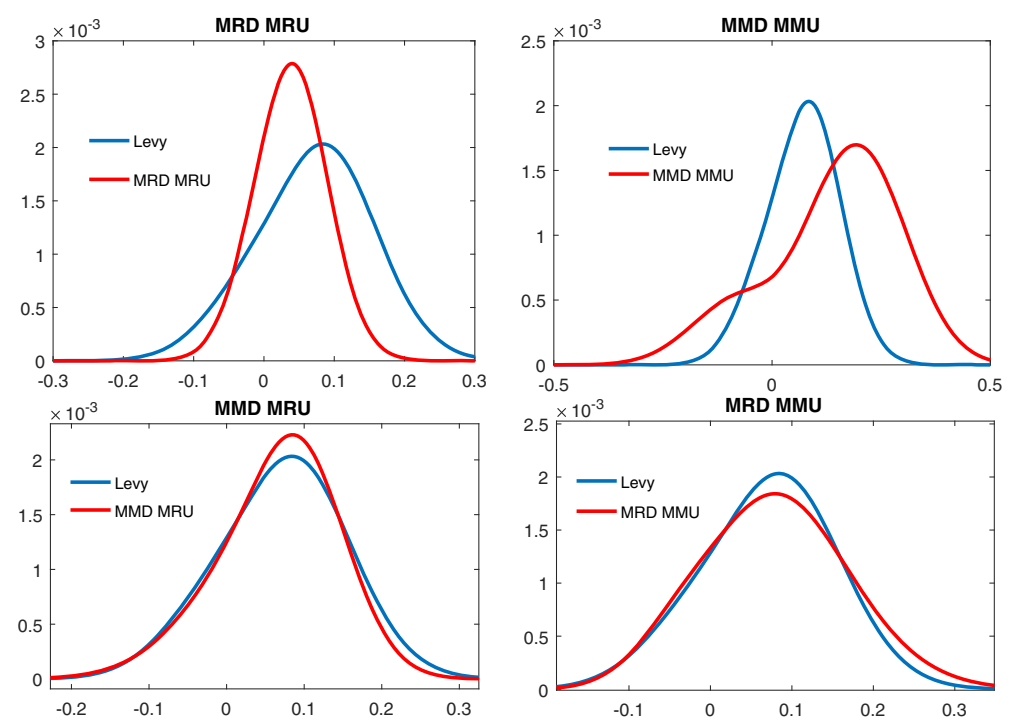

Fig. 2 Four Cases of Mean Reversion and Momentum on the Up and Down Sides

grid point. The grid is defined with respect to returns and not price levels and the return is always relative to the current stock price of the day.

For the grid, the current spot price is 100 and we employ a 1600 point non-uniform grid ranging from levels 50 to 200 for the spot price. In order to have a substantial variance for the diffusion approximation to the immediate neighbours, the parameters $C_{L}, C_{R}$ are scaled by a thousand. Therefore to build the density at, say, $n$ days we scale the generator by $n / 1000$ before evaluating its matrix exponential. The probability element is constructed at the 1600 return points on matrix exponentiation for one-, five-, and ten-day returns.

This probability element is used by interpolation to construct an unnormalized density evaluated on a uniform grid of 10,000 points ranging from the minimum to the maximum return on the original grid. The normalization factor is evaluated by numerical integration. The result is a density and a distribution function. The density is for used in maximum likelihood estimation and the distribution function is employed to evaluate digital tail probabilities for lower tails of negative returns and upper tails for positive returns. For maximum likelihood, the sum of the log likelihoods are evaluated for one-, five- and ten-day returns using overlapping data for the five and ten day returns. For digital moment estimation, the root mean square error between observed and model tail probabilities separately for one-, five- and ten-day returns are evaluated and then these three fit statistics are averaged. Digital moment estimation of parameters as developed and reported in Madan (2015b) gives a better fit to tail probabilities than maximum likelihood. We found this to be the case here as well and report results for just digital moment estimation. Figure 3 presents the fit to observed tail probabilities using digital 

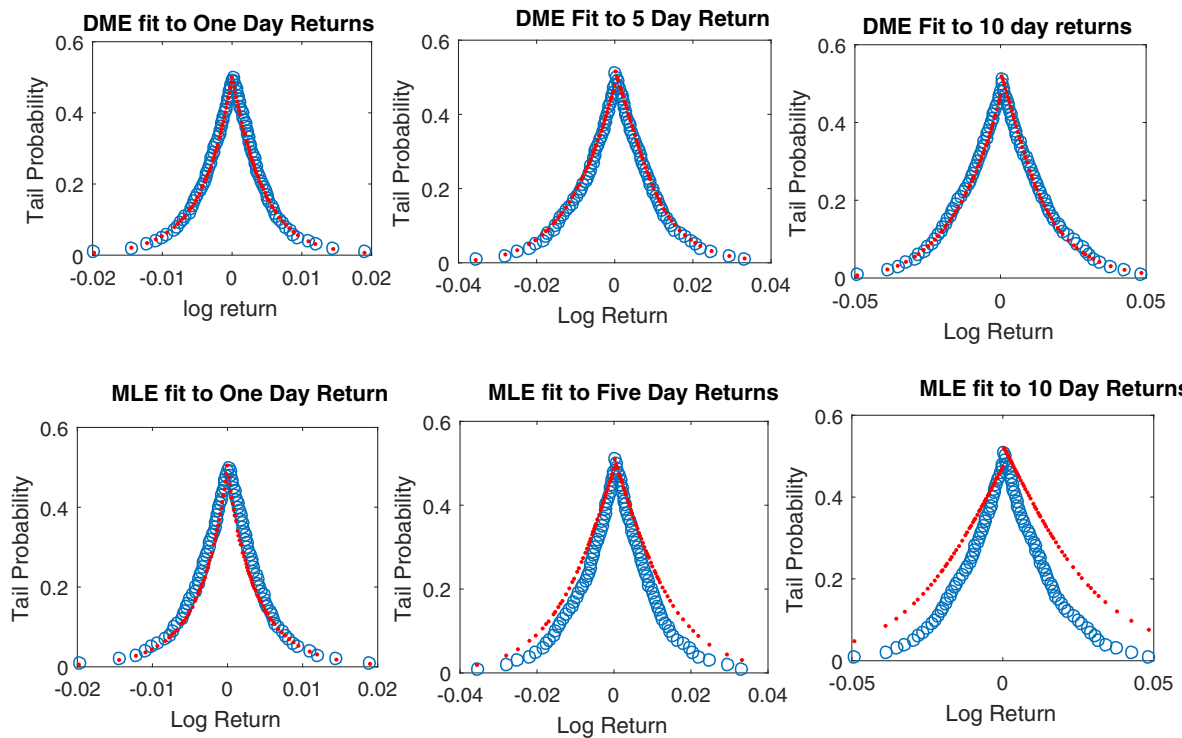

Fig. 3 Digital Moment and MLE fit to tail probabilities for one-, five- and ten-day returns. The observed probabilities are in circles while the estimated model probabilities are represented by dots

moments and maximum likelihood for the one case in which both estimations were conducted.

\section{Digital moment estimation results}

The spatially inhomogeneous variance gamma model was estimated for three underliers, one stock and two indexes, and two ratios of underliers. The stock ticker was $J P M$ and the two indices were the Exchange Traded Fund for the financial sector with ticker $X L F$ and the $S \& P 500$ index with ticker $S P X$. The two ratios were that of $J P M$ to $X L F$ and $X L F$ to $S P X$. In each case the 9 parameters of the model were estimated. Graphs of the fit of model digital tail probabilities to the observed tail probabilities are also presented. We present in separate subsections the graphs for the functions $C(X), G(X)$, and $M(X)$ and the graphs for the fit to tail probabilities. The data period ranged for a thousand days from August 2, 2012 to July 26, 2016.

\section{JPM}

In this case $G, M$ were independent of $X$, and Fig. 4 presents a graph of the function $C(X)$. The constant values for $G$ and $M$ were, respectively, 160 and 143 . The presence of leverage may be observed in the behaviour of $C$.

The fit to one-, five- and ten-day returns is presented in Fig. 5. 


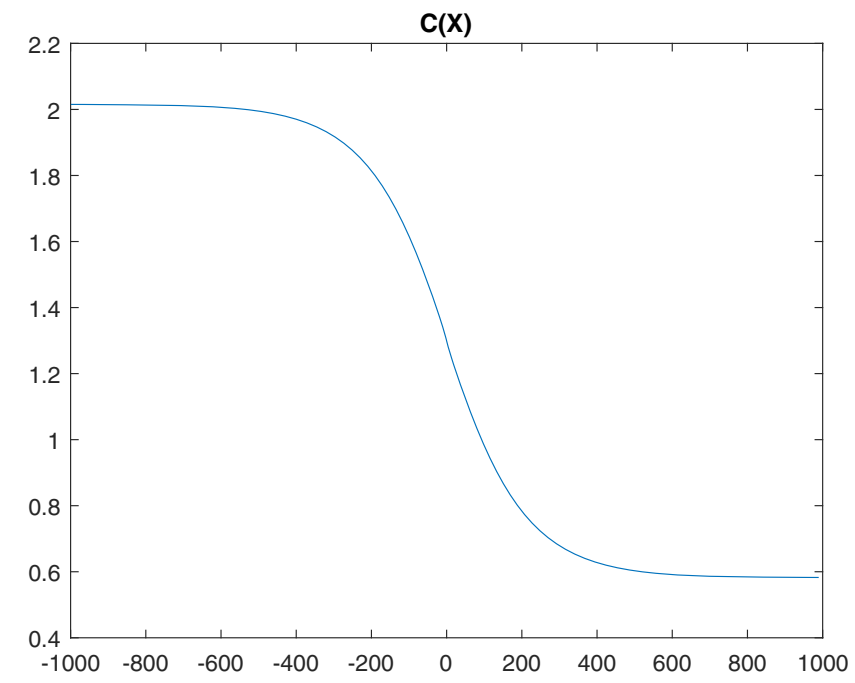

Fig. 4 The function $\mathrm{C}(\mathrm{X})$ for JPM

\section{XLF}

Figure 6 presents the parameters as functions of the log price relative. The behavior of $C$ reflects leverage while that of $M$ and $G$ suggest mean reversion upwards but momentum downwards in the structure of shocks. Figure 6 presents the function $C(X)$ over a much narrower range than Fig. 4 and focuses in on the behavior near zero as opposed to the tails.

Figure 7 presents the graph for the fit to one-, five- and ten-day tail probabilities.

\section{SPX}

Figure 8 presents the graphs for the parameters as functions of the log price relative. The general picture is similar to $X L F$ reflecting leverage in $C$, mean reversion upwards in $M$ and momentum down in $G$.

The graph for the fit to one-, five- and ten-day returns is presented in Fig. 9.
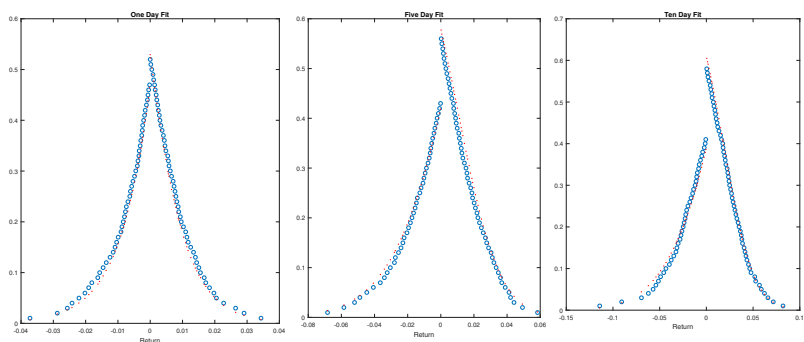

Fig. 5 Fit of spatially inhomogeneous variance gamma to one-, five- and ten-day returns on JPM. Observed tail probabilities in circles. Model probabilities shown by dots 

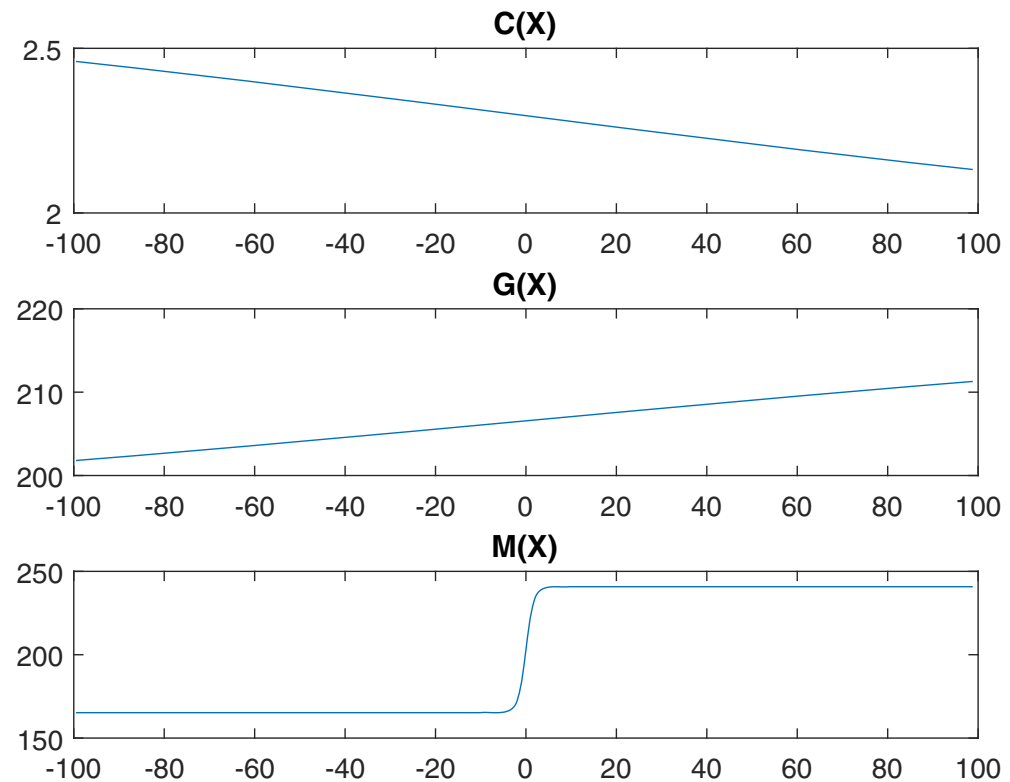

Fig. 6 The CGM parameters as functions of the log price relative for XLF

\section{JPM2XLF}

Figure 10 graphs the parameters as functions of the log price relative for the ratio of $J P M$ to $X L F$. This ratio reflects leverage in $C$, mean reversion down in $G$ but momentum upwards for $J P M$ relative to $X L F$.

The fit to one-, five- and ten-day returns is presented in Fig. 11.

\section{XLF2SPX}

Figure 12 presents the graphs for the parameters as functions of the log price relative. This ratio reflects a rise in volatility when $X L F$ rises relative to $S P X$ with mean reversion upwards and momentum downwards.
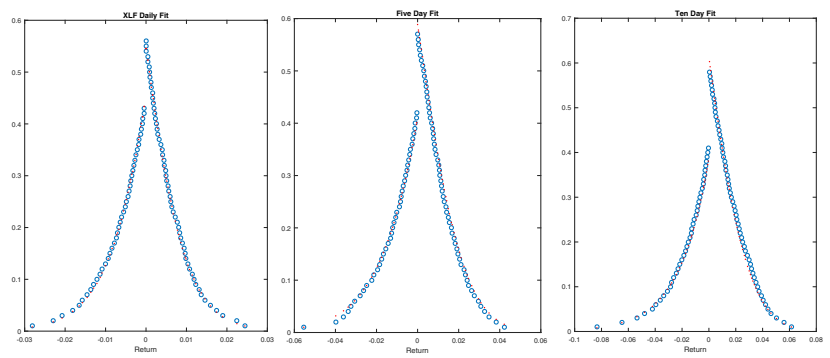

Fig. 7 Fit of spatially inhomogeneous variance gamma to one-, five- and ten-day returns on XLF. Observed tail probabilities in circles. Model probabilities shown by dots 

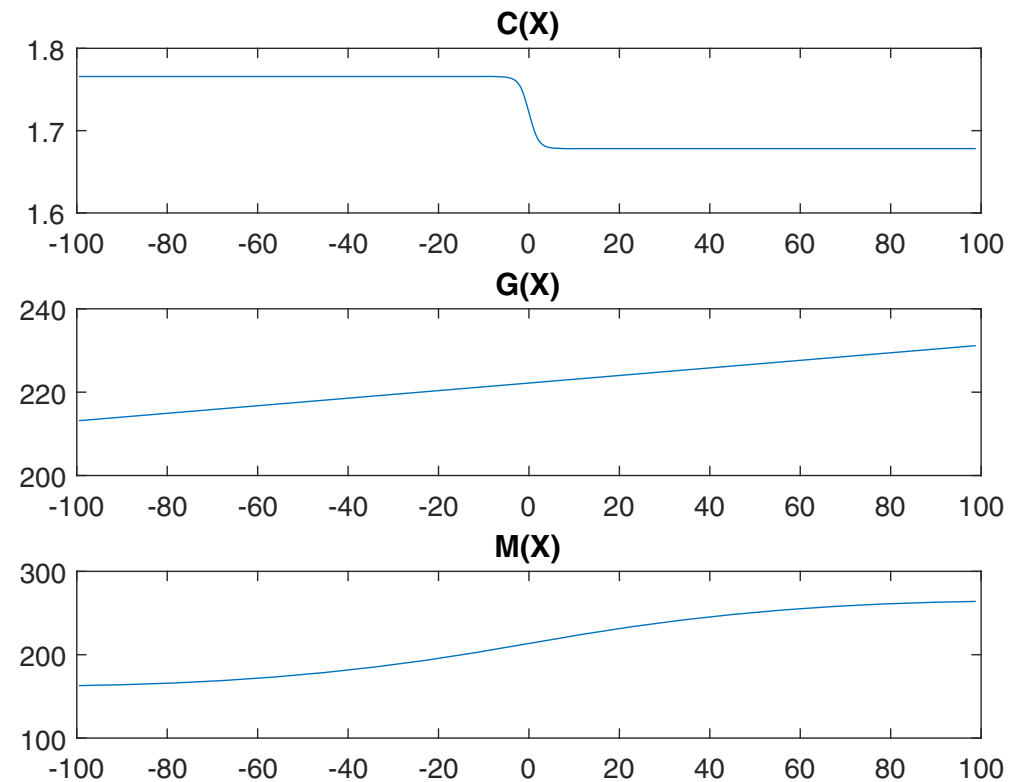

Fig. 8 Parameters as functions of the log price relative for SPX

The graphs for the fit to one-, five- and ten-day returns is presented in Fig. 13.

\section{Drift structure of SIVG}

The SIVG process inherits all its properties from the structure of the inhomogeneous jump compensator. If we ask, for example, whether there is a limiting stationary distribution, then we need to consider the evolution as described by Eq. (2). Suppose,
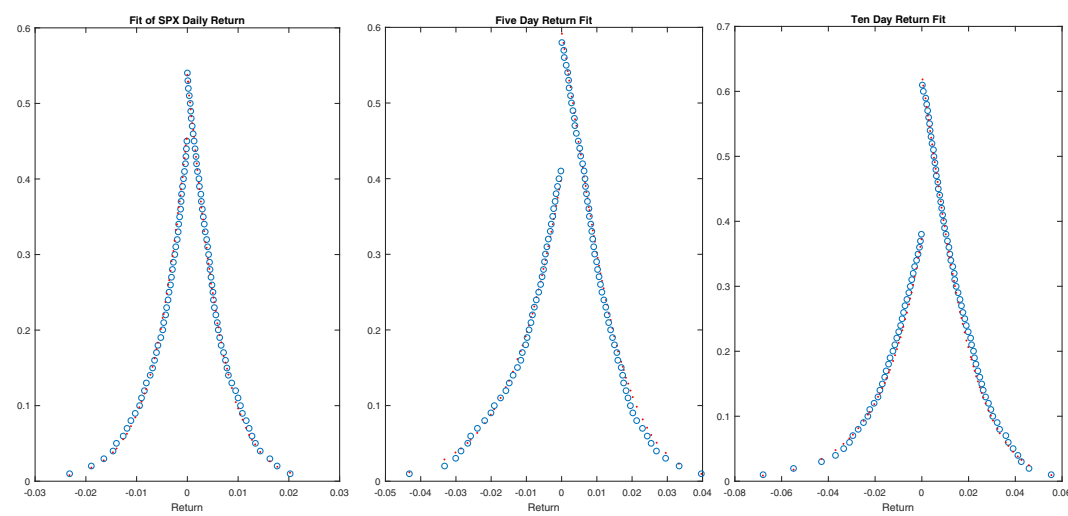

Fig. 9 Fit of spatially inhomogeneous variance gamma to one-, five- and ten-day returns on SPX. Observed tail probabilities in circles. Model probabilities shown by dots 

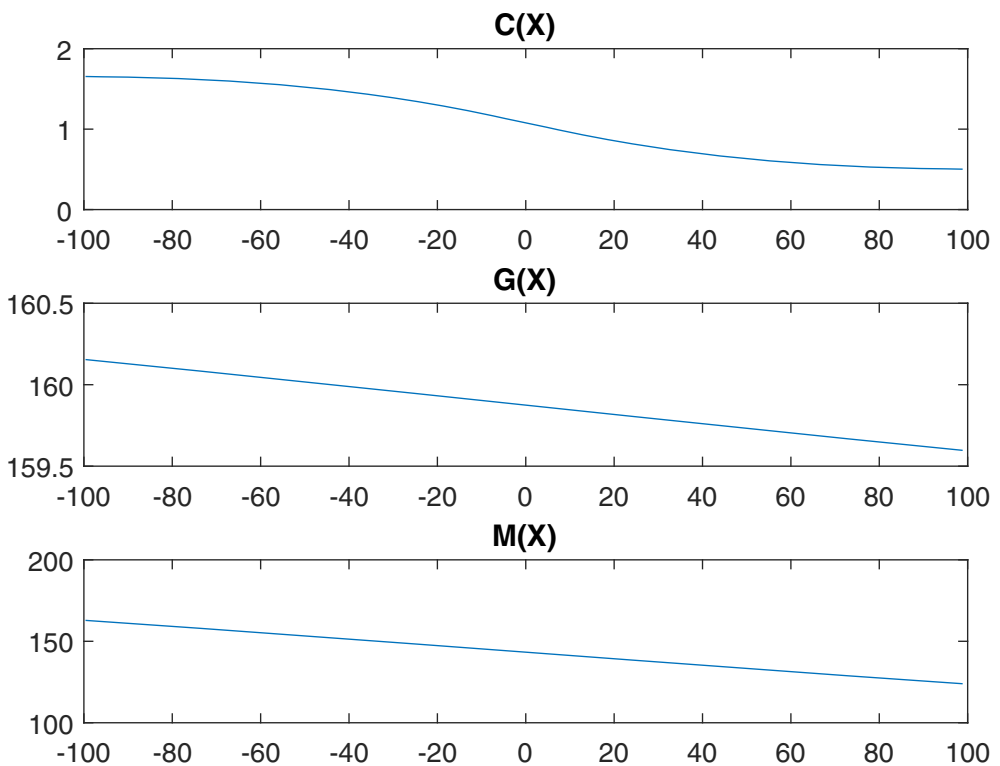

Fig. 10 Parameters as functions of the log price relative for the JPM XLF ratio

for example, we have $\delta(X) \equiv 0$, then $X(t)$ is a martingale with time independent compensator. We cannot then have any decrease in forward variances as would be required for convergence to a limiting stationary distribution. Hence the structure of $\delta(X)$ is critical for the answer to such questions. For the models estimated, on occasion, we find local mean reversion with $\delta(X)$ negatively sloped near zero. Over a wider range, however, there can be momentum effects with $\delta(X)>0$ for large positive $X$ and negative for large negative $X$. In some cases we may have global mean reversion with $\delta(X)>0$ for $X<0$ and the other way around for $X>0$.
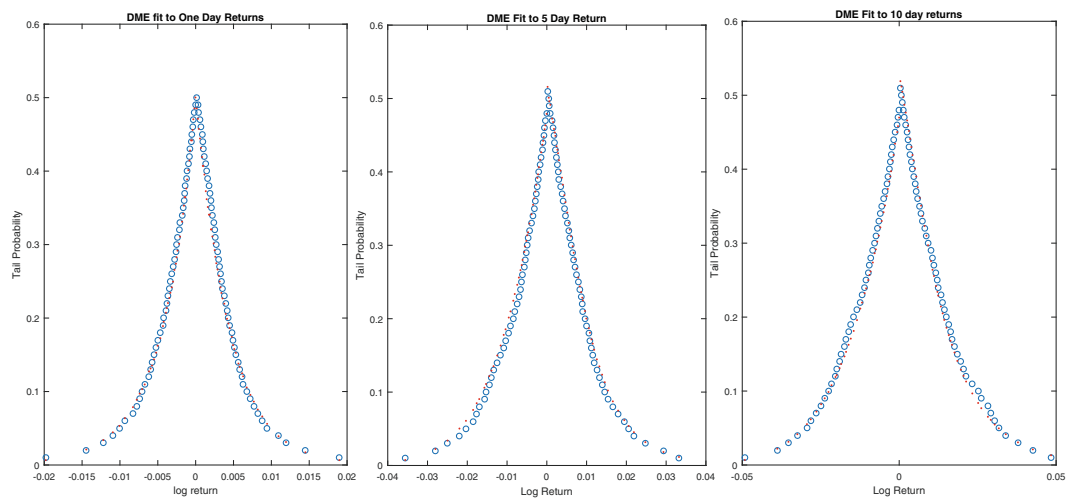

Fig. 11 Fit of spatially inhomogeneous variance gamma to one-, five- and ten-day returns on the JPM XLF ratio. Observed tail probabilities in circles. Model probabilities shown by dots 

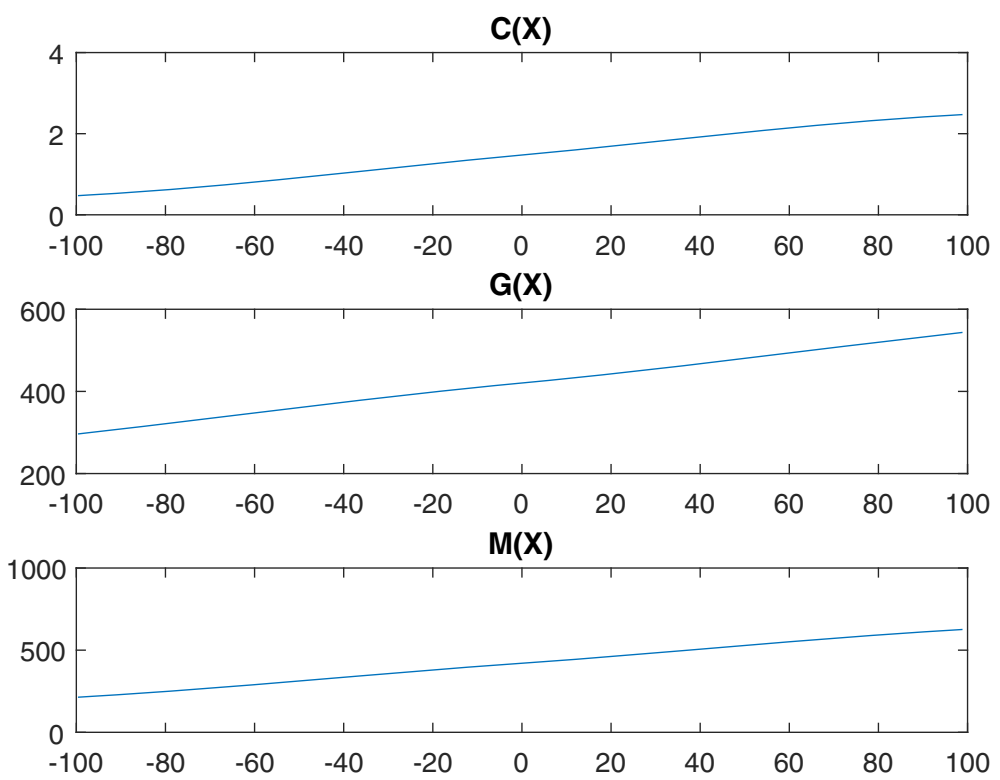

Fig. 12 Parameters as functions of the $\log$ price relative for the ratio of XLF to SPX

We comment here on the structure of local mean reversion especially if we replace $\delta(X)$ by its local linear approximation to get a non-Gaussian Ornstein-Uhlenbeck equation (Barndorff-Nielsen and Shephard 2001). Figure 14 presents the five estimated functions for $\delta(X)$ along with their local linear approximations. We observe that $J P M$ is estimated with mean reversion around an upward trend. $X L F, S P X$ and the ratio of $X L F$ to $S P X$ display local mean reversion with global momentum on both sides of the drift. The ratio of $J P M$ to $X L F$ has drift momentum on both sides.
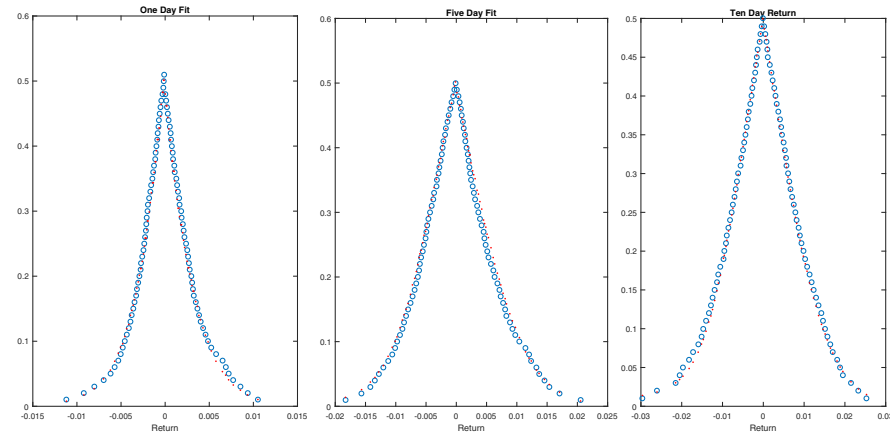

Fig. 13 Fit of spatially inhomogeneous variance gamma to one-, five- and ten-day returns on the XLF SPX ratio. Observed tail probabilities in circles. Model probabilities shown by dots 

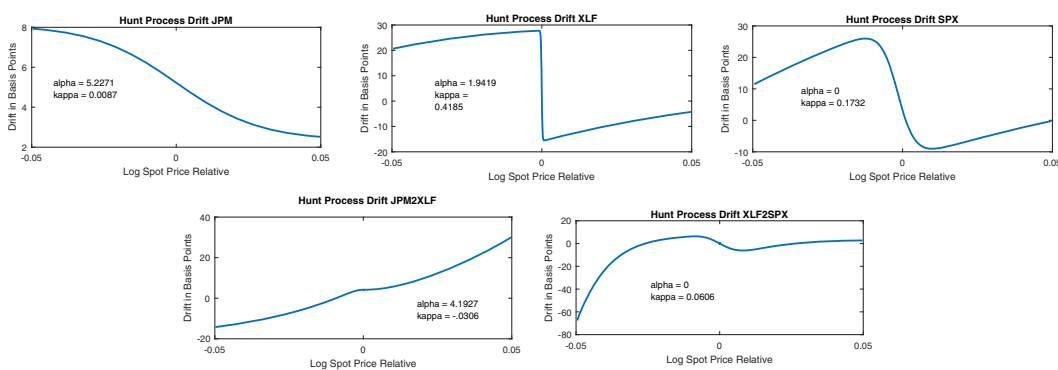

Fig. 14 The function $\delta(X)$ and linear approximations. $\alpha$ is the intercept and $\kappa$, when positive, is the rate of mean reversion

\section{Mean reversion and momentum in the process}

We evaluate the ratio of the conditional probability for an $x \%$ move or greater, down and up in the next five days, conditional on an $x \%$ move down or up just having occurred to the unconditional probability for such a move. If the conditional probability of an up move rises relative to its unconditional probability, then we have momentum upwards. Similarly for a down move. If the probabilities of down moves rise conditional on an up move, then we have mean reversion.

Figure 15 presents the ratios of conditional tail probabilities to their unconditional probabilities. The conditional probabilities condition on up and down moves in five days of 50 and 100 basis points for the five estimated models.

We observe, by and large, the presence of momentum. For the ratio of $X L F$ to $S P X$ the probabilities of large up moves rise on a down move fall on an up move. Hence there is some mean reversion conditional on large moves in this case. The other cases all support a momentum hypothesis with up and down probabilities rising and the down and up probabilities falling with an up and down move.
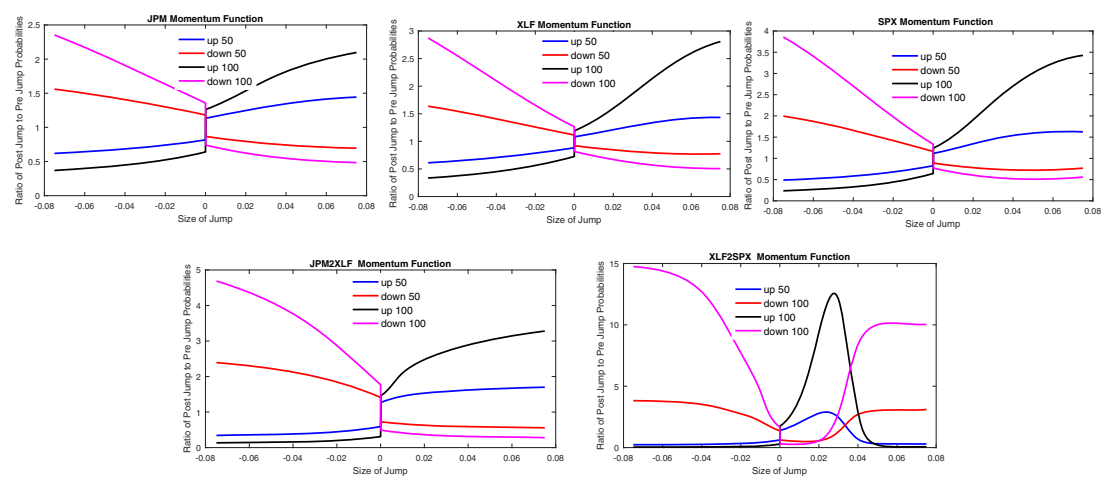

Fig. 15 Ratios of conditional tail probabilities relative to their unconditional counterparts for the five estimated models 


\section{Risk compensation}

Classically, the motion of a stock has been described as a random variable over some horizon with a mean and some volatility. Risk compensation then relates attributes of the random component to the mean return. At the level of surprise moves captured in the arrival rate function there is no mean return to be explained and neither is there a volatility. We only have the arrival rate function. Madan and Schoutens (2016b) shows and the introduction summarized the reward concept to be derived from the arrival rate function as the exponential variation. This is the limiting drift rate for the process that now depends on the spatial variable for a spatially inhomogeneous process. We may then ask what the risk is and how and whether the reward is related to this risk.

In economies satisfying the law of one price, there is a price to be explained and rewards are explained by covariations of returns with the change of measure from probability to price. All events are viewed as having both a price and a probability with a change of measure essentially given by the ratio of the two. It then follows algebraically that excess returns may be related to the covariation of returns with the measure change. Different asset pricing models propose different and diverse candidate models for the measure change (see Skiadis (2009) for example).

With financial markets taken to be in a state of continuous and surprising motion the law of one price is suspended as the price is always in a state of flux. Transactions occur at a myriad of prices arising out of negotiations in the process of price discovery. We go on instead to consider the possibility of coupling no arbitrage with the fiction of a two price economy, replacing the fiction of the law of one price. In a one price economy the terms of trade are invariant to the direction of trade as one may buy or sell any amount at the going price. Hence zero cost cash flows or cash flows net of their prices form a subspace closed under negation. For two price economies the terms of trade vary with the direction of trade with purchases from market occuring at higher prices than sales to the market. The set of zero cost cash flows remains a convex cone but is no longer closed under negation and hence is not a subspace. Under no arbitrage it fails to meet the nonnegative cash flows and convex set separation principles yield a class of measures under which the zero cost cash flows have a nonpositive expectation (see Madan 2015a).

Describing acceptable risks as those with a positive expectation under a set of test measures in keeping with the structure of such risks in Artzner et al. (1999) the upper and lower prices of the two price economy are seen as suprema and infima of expectations under a suitable set of test measures. Modeling the test measures as those with event probabilities bounded above by a probability distortion of the physical or true probability renders the upper and lower prices to be distorted expectations as presented for example in Kusuoka (2001). One merely composes the distribution function of the random variable to be evaluated with a concave distribution function on the unit interval to form a distorted distribution function, before taking expectations. The result is a distorted expectation. Passing to the continuous time limit one replaces probability distortions by measure distortions of arrival rate functions (Madan et al. 2016). 
Madan and Schoutens (2016b) investigate the relationship in the economy between exponential variations as reward related to two sided risk concepts based on the shave in value embedded in the lower price and the add-on to value embedded in the upper price. Madan and Schoutens (2016b) show that exponential variations as rewards reflect premia for both of the two sided risk concepts embedded in two price economies. Hence covariation based risk exposures and premia are replaced by explicit risk charges related to the risk of price fluctuations as assessed by the lower and upper prices existing in two price economies.

Here this very same relationship is investigated for the five estimated processes separately, relating spatially contingent exponential variations to the two sided spatially contingent risk measures of the lower price shave and the upper price add-on. For the lower and upper price construction one needs to specify the measure distortions. These are the same distortions as employed in the Madan and Schoutens (2016b) study. These distortions were calibrated to bring risk neutral prices embedded in option markets to lie within the measure distorted physical arrival rates for data on the $S \& P 500$ index. Both the option and time series data on index options were employed for the calibration.

The measure distortions are two increasing concave/convex functions of the positive half line that are bounded below/above by the identity. The specific parametric distortions employed are

$$
\begin{aligned}
& \Gamma^{+}(x)=x+a\left(1-e^{-c x}\right)^{\frac{1}{1+\gamma}} \\
& \Gamma^{-}(x)=x-\frac{b}{c}\left(1-e^{-c x}\right) .
\end{aligned}
$$

The values for $a, b, c$, and $\gamma$ are $3.1191,0.9694,0.3108$, and 0.4877 . For an arrival rate function $k(X, x)$ define the measure of a measurable subset $A$ of the real line by

$$
K(X, A)=\int_{A} k(X, x) d x
$$

Now the lower price $L$, the exponential variation $V$, and the upper price $U$ may be written as

$$
\begin{aligned}
& L=-\int_{0}^{\infty} \Gamma^{+}\left(K\left(X,\left[\left(e^{x}-1\right)^{-}>v\right]\right)\right) d v+\int_{0}^{\infty} \Gamma^{-}\left(K\left(X,\left[\left(e^{x}-1\right)^{+}>v\right]\right)\right) d v \\
& V=-\int_{0}^{\infty} K\left(X,\left[\left(e^{x}-1\right)^{-}>v\right]\right) d v+\int_{0}^{\infty} K\left(X,\left[\left(e^{x}-1\right)^{+}>v\right]\right) d v \\
& U=-\int_{0}^{\infty} \Gamma^{-}\left(K\left(X,\left[\left(e^{x}-1\right)^{-}>v\right]\right)\right) d v+\int_{0}^{\infty} \Gamma^{+}\left(K\left(X,\left[\left(e^{x}-1\right)^{+}>v\right]\right)\right) d v .
\end{aligned}
$$

So the risk charge for the shave to lower price is

$$
-L+V
$$


Also the upper price add-on is

$$
U-V=\int_{0}^{\infty}\left(I-\Gamma^{-}\right) \circ\left(K\left(X,\left[\left(e^{x}-1\right)^{-}>v\right]\right)\right) d v+\int_{0}^{\infty}\left(\Gamma^{+}-I\right) \circ\left(K\left(X,\left[\left(e^{x}-1\right)^{+}>v\right]\right)\right) d v .
$$

If the variation is related to the risk charges with premia $\alpha, \beta$ then

$$
V=\alpha(V-L)+\beta(U-V) .
$$

As a consequence,

$$
V=\frac{\beta U-\alpha L}{1+(\beta-\alpha)}
$$

Therefore, a relationship is sought between $V, U, L$. Conditional on $X$, the regression

$$
V(X)=a+b L(X)+c U(X),
$$

is estimated. The estimated values for $a$ were near zero and then solving for $\alpha, \beta$ yields

$$
\begin{aligned}
& \alpha=-\frac{b}{1-(b+c)} \\
& \beta=\frac{c}{1-(b+c)} .
\end{aligned}
$$

Table 1 provides the values for $\alpha, \beta$ for the five estimated processes.

All the estimated SIVG processes have rewards linearly related to risk as embodied in the lower and upper prices and this is the case whether we work with assets or their ratios. The coefficients reflect the response of exponential variations to tail-risk on both sides. A positive coefficient on the lower price suggests that a rise in the down tail attains return compensation suggestive of mean reversion on the down side. A negative coefficient lowers future returns suggestive of momentum on the down side. Similarly, a negative coefficient on the upper price represents mean reversion on the up side while a positive coefficient reflects momentum on the up side.

\section{The S\&P 500 index during the financial crisis}

We report here on the SIVG model fitted for data on the S\&P 500 index for the years 2007 through 2009. Figure 16 presents a graph of the dependence of the parameters

Table 1 Premia for lower shave and upper add-on

\begin{tabular}{llc}
\hline Asset & $\alpha$ & $\beta$ \\
\hline JPM & 3.3013 & -3.2174 \\
XLF & -3.9919 & 3.9366 \\
SPX & -2.5735 & 2.7636 \\
JPM/XLF & -0.7864 & 0.6773 \\
XLF/SPX & -0.5514 & 0.4355 \\
\hline
\end{tabular}



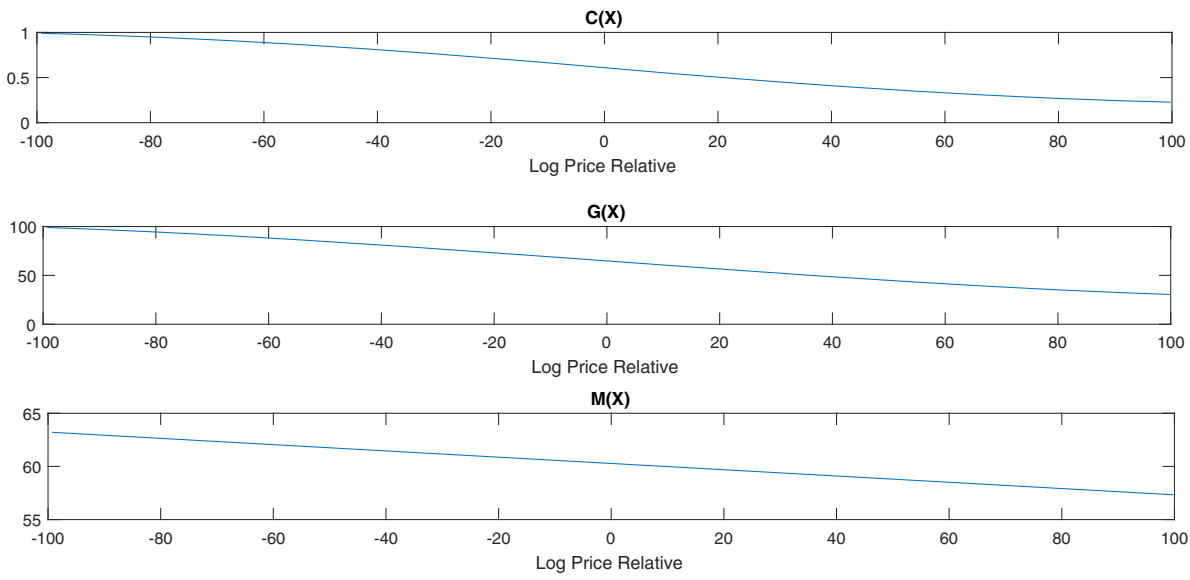

Fig. 16 Graphs for the spatial dependence of C, G, and M for the period 2007 to 2009

$C, G$, and $M$ on the logarithm of the price relative. We observe the presence of leverage, mean reversion on the down side, and momentum upwards.

The graphs for the fit to one, five and ten day returns are presented in Fig. 17.

Figure 18 graphs the drift as a function of the spatial variate presented as a return. Mean reversion is present locally.

Figure 19 presents the momentum function. The momentum though positive is considerably reduced when compared to the non-crisis period.

Finally, we estimated the up and down side risk premia, and they were both positive suggesting a downside mean reversion coupled with an upward momentum. The values for $\alpha$, and $\beta$ were, respectively, 0.4657 and 0.0012 .

\section{Conclusion}

The dynamics of movements in prices are modeled as the accumulation of surprise jumps in the logarithm. As a consequence, there are no deterministic time components or any continuous martingale components. All the information about price changes is embedded in the arrival rate function compensating the surprise jumps.
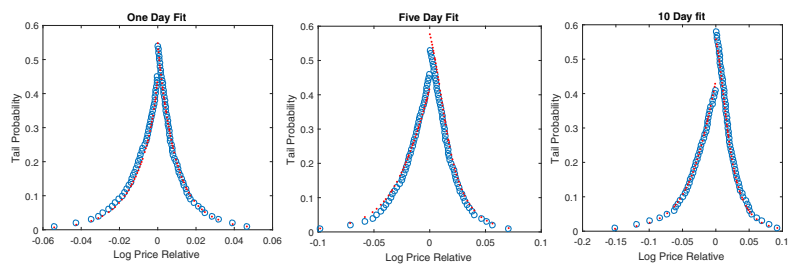

Fig. 17 The fit to digital tail probabilities for one-, five- and ten-day returns 


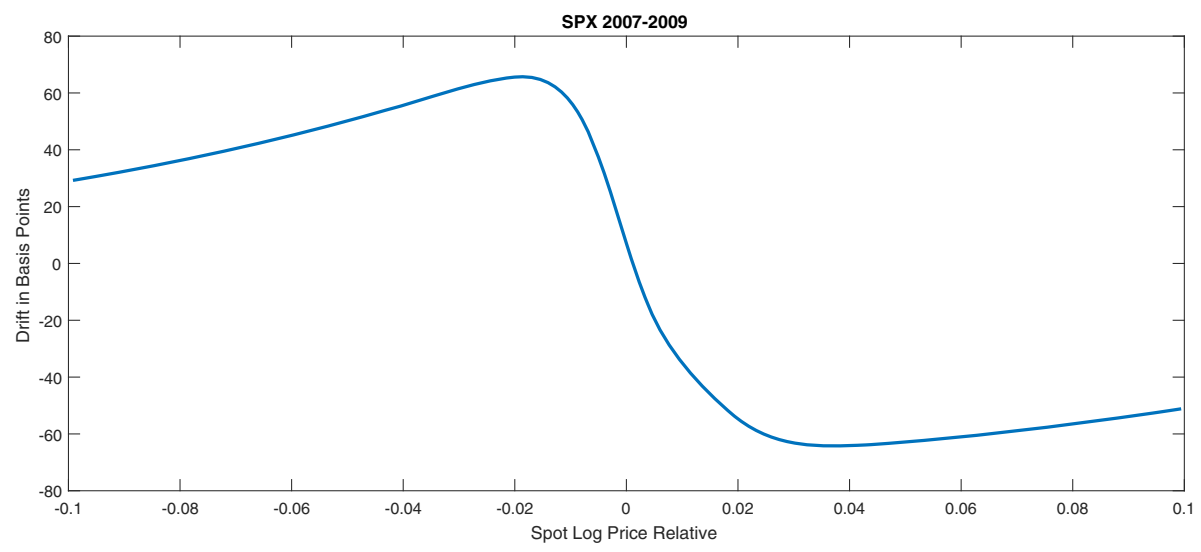

Fig. 18 The drift as a function of $X$

A Markov pure jump model is formulated for the motion by making the parameters of the variance gamma Lévy measure deterministic functions of the level of prices. Estimation is conducted by employing a continuous-time finite-state Markov chain approximation that yields probability elements on matrix exponentiation of the transition rate matrix. The dynamics is then decomposed into a space dependent drift and a space dependent martingale component. Though there is some local mean reversion, by and large, the dynamics estimated is that of the momentum type. Risk compensation is estimated via a linear relation between the exponential variation and measure distorted variations for the lower and upper prices of two-price economies. Estimations are conducted for the S\&P 500 index (SPX), the exchange traded fund for the financial sector (XLF), J. P. Morgan stock prices (JPM), the ratio of JPM to XLF, and the ratio of XLF to SPX.

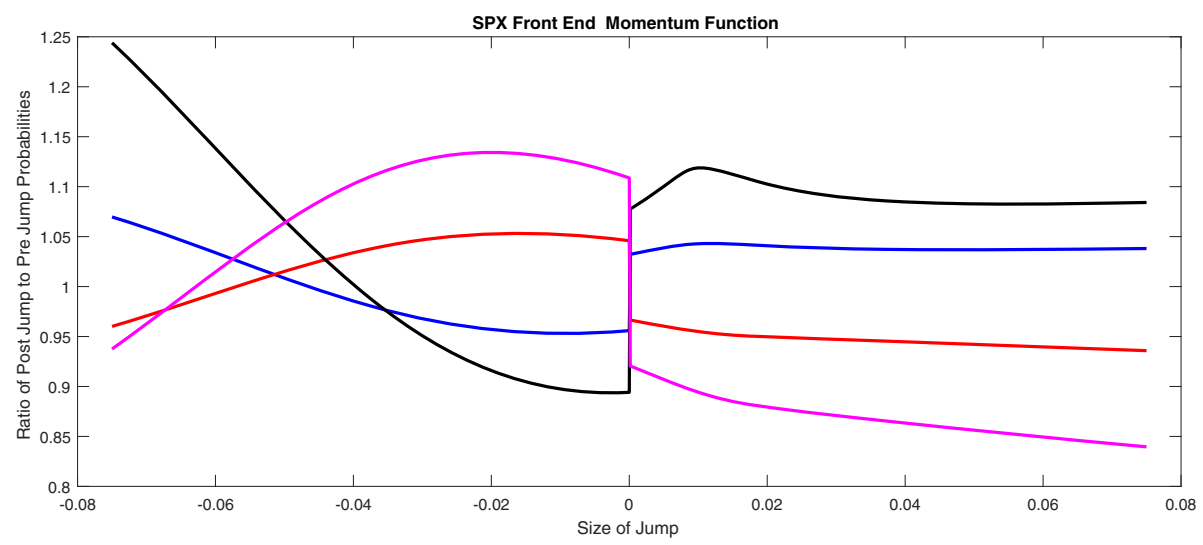

Fig. 19 Momentum function for the period 2007 to 2009 


\section{Competing interests}

The author declares that he/she has no competing interests.

\section{References}

Angelos, B: The Hunt Variance Gamma Process with Applications to Option Pricing. University of Maryland, PhD. Dissertation (2013)

Artzner, P, Delbaen, F, Eber, M, Heath, D: Coherent Measures of Risk. Math. Finance 9, 203-228 (1999)

Barndorff-Nielsen, O, Shephard, N: Non-Gaussian Ornstein-Uhlenbeck-based models and some of their uses in financial economics (with discussion). J. R. Stat. Soc. Ser. B 63, 167-241 (2001)

Bass, RF: Uniqueness in law for pure jump Markov processes. Probab. Theory Relat. Fields 79, 271-287 (1988)

Carr, P, Geman, H, Madan, D, Yor, M: The fine structure of asset returns: An empirical investigation. J. Bus 75(2), 305-332 (2002)

Cherny, A, Madan, DB: Markets as a counterparty: An Introduction to Conic Finance. Int. J. Theor. Appl. Finance 13, 1149-1177 (2010)

Cont, R, Minca, A: Recovering Portfolio Default Intensities Implied by CDO Quotes. Math. Finance 23, 94-121 (2013)

Cousin, A, Crépey, S, Kan, YH: Delta-hedging correlation risk. Rev. Deriv. Res 15, 25-56 (2012)

Delbaen, F, Schachermayer, W: A general version of the fundamental theorem of asset pricing. Math. Ann 300, 463-520 (1994)

Hunt, G: Martingales et Processus de Markov. Dunod, Paris (1966)

Khintchine, AY: Limit laws of sums of independent random variables. ONTI, Moscow, Russian (1938)

Kusuoka, S: On Law Invariant Coherent Risk Measures. Adv. Math. Econ 3, 83-95 (2001)

Lévy, P: Théorie de l'Addition des Variables Aléatoires. Gauthier-Villars, Paris (1937)

Madan, DB: Asset pricing theory for two price economies. Ann. Finance 11, 1-35 (2015a)

Madan, DB: Estimating parametric models of probability distributions. Methodol. Comput. Appl. Probab 17, 823-831 (2015b)

Madan, DB, Schoutens, W: Applied Conic Finance. Cambridge University Press, Cambridge (2016a)

Madan, DB, Schoutens, W: Conic Asset Pricing and The Costs of Price Fluctuations (2016b). Working Paper, Robert H. Smith School of Business

Madan, DB: Instantaneous Portfolio Theory (2016a). Available at https://ssrn.com/abstract=2804718

Madan, DB: Momentum and reversion in risk neutral martingale probabilities. Quant. Finan 14, 777-787 (2016b). Available at https://ssrn.com/abstract=2251300

Madan, DB: Risk Premia in Option Markets. Ann. Finance 12, 71-94 (2016c)

Madan, D, Carr, P, Chang, E: The variance gamma process and option pricing. Eur Financ Rev 2, 79-105 (1998)

Madan, DB, Pistorius, M, Stadje, M: On Dynamic Spectral Risk Measures and a Limit Theorem (2016). forthcoming Finance and Stochastics

Madan, DB, Seneta, E: The variance gamma (VG) model for share market returns. J. Bus 63, 511-524 (1990)

Pistorius, M, Mijatović, A: Continuously Monitored Barrier Options under Markov Processes. Math. Finance 23, 1-38 (2011). Also available at https://ssrn.com/abstract=1462822

Sato, K: Lévy Processes and Infinitely Divisible Distributions. Cambridge University Press, Cambridge (1999)

Skiadis, C: Asset Pricing Theory. Princeton University Press, Princeton (2009)

Stroock, DW: Diffusion processes associated with L évy generators. Probab. Theory Relat. Fields 32, 209_ 244 (1975)

Stroock, DW, Varadhan, SRS: Multidimensional diffusion processes, a series of comprehensive studies in mathematics. vol. 233. Springer-Verlag, Berlin (1979) 